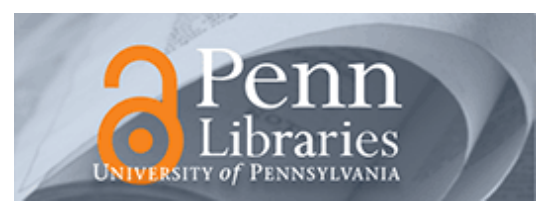

University of Pennsylvania

ScholarlyCommons

May 2005

\title{
Generalized optical theorem for reflection, transmission, and extinction of power for electromagnetic fields
}

D. R. Lytle II

University of Illinois

P. Scott Carney

University of Illinois

John C. Schotland

University of Pennsylvania, schotland@seas.upenn.edu

Emil Wolf

University of Rochester

Follow this and additional works at: https://repository.upenn.edu/be_papers

\section{Recommended Citation}

Lytle II, D. R., Carney, P. S., Schotland, J. C., \& Wolf, E. (2005). Generalized optical theorem for reflection, transmission, and extinction of power for electromagnetic fields. Retrieved from

https://repository.upenn.edu/be_papers/70

Copyright American Physical Society. Reprinted from Physical Review E, Volume 71, Issue 5, Article 056610, May 2005, 8 pages.

Publisher URL: http://dx.doi.org/10.1103/PhysRevE.71.056610

This paper is posted at ScholarlyCommons. https://repository.upenn.edu/be_papers/70

For more information, please contact repository@pobox.upenn.edu. 


\title{
Generalized optical theorem for reflection, transmission, and extinction of power for electromagnetic fields
}

\author{
Abstract \\ We present a generalization of the optical theorem for electromagnetic fields. This result is used to obtain \\ the power extinguished from a field by a scatterer contained in a dielectric half space. Applications to \\ microscopy and tomography are described. \\ Comments \\ Copyright American Physical Society. Reprinted from Physical Review E, Volume 71, Issue 5, Article \\ 056610, May 2005, 8 pages. \\ Publisher URL: http://dx.doi.org/10.1103/PhysRevE.71.056610
}




\title{
Generalized optical theorem for reflection, transmission, and extinction of power for electromagnetic fields
}

\author{
D. R. Lytle II \\ Department of Electrical and Computer Engineering, University of Illinois at Urbana-Champaign, Urbana, Illinois 61801, USA \\ P. Scott Carney \\ Department of Electrical and Computer Engineering and the Beckman Institute for Advanced Science and Technology, University of \\ Illinois at Urbana-Champaign, Urbana, Illinois 61801, USA \\ John C. Schotland \\ Department of Bioengineering, University of Pennsylvania, Philadelphia, Pennsylvania 19104, USA \\ Emil Wolf \\ Department of Physics and Astronomy and the Institute of Optics, University of Rochester, Rochester, New York 14627, USA and The \\ College of Optics and Photonics/CREOL, University of Central Florida, Orlando, Florida 32816, USA
}

(Received 12 November 2004; published 20 May 2005)

\begin{abstract}
We present a generalization of the optical theorem for electromagnetic fields. This result is used to obtain the power extinguished from a field by a scatterer contained in a dielectric half space. Applications to microscopy and tomography are described.
\end{abstract}

DOI: 10.1103/PhysRevE.71.056610

PACS number(s): 42.25.- $\mathrm{p}, 42.40 .-\mathrm{i}$

\section{INTRODUCTION}

The conservation of energy for electromagnetic fields leads to a remarkable identity known as the optical theorem. This theorem relates the power extinguished from a plane wave incident on an object to the scattering amplitude in the direction of the incident field. The optical theorem may be expressed by the formula [1-3]

$$
\sigma_{e}=\frac{\omega}{2|\mathbf{e}|^{2}} \operatorname{Im}\left[e_{\alpha} e_{\beta}^{*} A_{\alpha \beta}(\mathbf{k}, \mathbf{k})\right],
$$

where $\sigma_{e}$ is the extinction cross section, $\mathbf{e}$ is the complex vector amplitude of the incident field, and $A_{\alpha \beta}(\mathbf{k}, \mathbf{k})$ is the tensor scattering amplitude in the direction of the incident beam and the summation convention is assumed.

In this paper, we present a generalization of the optical theorem for elecromagnetic fields. We derive expressions for the extinguished power that are applicable to scatterers in free space as well as scatterers in a half space. These results take into account the contribution not only of the homogeneous components but also of the evanescent components of the incident field, both of which are necessary when the source of illumination is in the near zone of the scatterer. Results along these lines for scalar fields were recently presented [4].

The paper is organized as follows. In Sec. II, we derive an expression for the extinguished power that is the progenitor of the generalized optical theorem for electromagnetic fields. An expression is obtained that relates the extinguished power to a volume integral over the domain of the scatterer. This formulation also applies to the case that the scattering object is embedded in an arbitrary, inhomogeneous background. In Sec. III, scattering from an object in free space is analyzed. In Sec. IV, the problem of scattering from an object in a half space is addressed. It is found that the extinguished power is related to the field that is scattered in the directions of the components of the incident field in both the upper and the lower half spaces (see Fig. 2). The half space problem is of practical importance in applications to optical imaging when the sample is supported on a slide or other flat substrate.

\section{GENERAL RESULTS}

The conservation of energy in electromagnetic fields may be expressed by means of the Poynting vector [3, Sec. 1.4]. The time averaged Poynting vector for a fixed frequency, $\mathbf{S}(\mathbf{r})$, is given by the expression

$$
\mathbf{S}(\mathbf{r})=\frac{c}{8 \pi} \operatorname{Re}\left[\mathbf{E}(\mathbf{r}) \times \mathbf{H}^{*}(\mathbf{r})\right]
$$

where $\mathbf{E}(\mathbf{r})$ and $\mathbf{H}(\mathbf{r})$ the space dependent parts of the time varying fields, $\mathbf{E}(\mathbf{r}, t)=\operatorname{Re}\left[\mathbf{E}(\mathbf{r}) e^{-i \omega t}\right]$ and $\mathbf{H}(\mathbf{r}, t)=\operatorname{Re}\left[\mathbf{H}(\mathbf{r}) e^{-\mathrm{i} \omega t}\right]$. In a linear medium at fixed frequency $\omega=c k_{0}, \mathbf{E}(\mathbf{r})$ and $\mathbf{H}(\mathbf{r})$ satisfy the time independent form of the Maxwell equations which, in the Gaussian system of units, are

$$
\begin{gathered}
\boldsymbol{\nabla} \times \mathbf{H}(\mathbf{r})+i k_{0} \epsilon(\mathbf{r}) \mathbf{E}(\mathbf{r})=\mathbf{0}, \\
\boldsymbol{\nabla} \times \mathbf{E}(\mathbf{r})-i k_{0} \mu(\mathbf{r}) \mathbf{H}(\mathbf{r})=\mathbf{0} .
\end{gathered}
$$

Here $\epsilon(\mathbf{r})$ is the electric permittivity and $\mu(\mathbf{r})$ is the magnetic permeability. The fields can be expressed as the sum of the incident field $\mathbf{E}^{(i)}(\mathbf{r})$ and the scattered field $\mathbf{E}^{(s)}(\mathbf{r})$,

$$
\mathbf{E}(\mathbf{r})=\mathbf{E}^{(i)}(\mathbf{r})+\mathbf{E}^{(s)}(\mathbf{r})
$$




$$
\mathbf{H}(\mathbf{r})=\mathbf{H}^{(i)}(\mathbf{r})+\mathbf{H}^{(s)}(\mathbf{r}) .
$$

The incident field is assumed to be generated by sources outside the domain being considered and satisfies the freespace, time independent Maxwell equations

$$
\begin{aligned}
& \boldsymbol{\nabla} \times \mathbf{H}^{(i)}(\mathbf{r})+i k(\mathbf{r}) \mathbf{E}^{(i)}(\mathbf{r})=\mathbf{0}, \\
& \boldsymbol{\nabla} \times \mathbf{E}^{(i)}(\mathbf{r})-i k(\mathbf{r}) \mathbf{H}^{(i)}(\mathbf{r})=\mathbf{0},
\end{aligned}
$$

in addition to the fields being divergence free. The scattered field satisfies the equations

$$
\begin{gathered}
\boldsymbol{\nabla} \times \mathbf{H}^{(s)}(\mathbf{r})+i k(\mathbf{r}) \mathbf{E}^{(s)}(\mathbf{r})=-4 \pi i k(\mathbf{r}) \eta(\mathbf{r}) \mathbf{E}(\mathbf{r}) \\
\boldsymbol{\nabla} \times \mathbf{E}^{(s)}(\mathbf{r})-i k(\mathbf{r}) \mathbf{H}^{(s)}(\mathbf{r})=4 \pi i k(\mathbf{r}) \chi(\mathbf{r}) \mathbf{H}(\mathbf{r}),
\end{gathered}
$$

where $\eta(\mathbf{r})$ and $\chi(\mathbf{r})$ are the electric and magnetic susceptibilities of the scatterer, respectively, and $k(\mathbf{r})=k_{0} \sqrt{\mu \epsilon}$ is the wave number. The susceptibilities are related to the electric permittivity and the magnetic permeability by the formulas

$$
\begin{aligned}
& \boldsymbol{\epsilon}(\mathbf{r})=\frac{k(\mathbf{r})}{4 \pi k_{0}}[\eta(\mathbf{r})-1], \\
& \mu(\mathbf{r})=\frac{k(\mathbf{r})}{4 \pi k_{0}}[\chi(\mathbf{r})-1] .
\end{aligned}
$$

The distinction between the scatterer, characterized by $\eta(\mathbf{r})$ and $\chi(\mathbf{r})$, and the background, characterized by $k(\mathbf{r})$ is arbitrary. That is, $k(\mathbf{r}), \eta(\mathbf{r})$, and $\chi(\mathbf{r})$ may be redefined in any way such that $\epsilon(\mathbf{r})$ and $\mu(\mathbf{r})$ appearing in Eqs. (3) and (4) remain unchanged. In many cases of interest there are compelling reasons to distinguish a scatterer from the background. In free space $k(\mathbf{r})=k_{0}$ everywhere. Because we assume that the background medium is lossless, the power absorbed by the scatterer, $P_{a}$, is given by the integral of the Poynting vector passing through any surface enclosing the scatterer but not the sources of the incident field:

$$
P_{a}=-\int_{\partial V} d^{2} r \mathbf{S}(\mathbf{r}) \cdot \hat{\mathbf{n}}
$$

Here $\hat{\mathbf{n}}$ is the outward unit normal to the surface $\partial V$ of the scatterer. Making use of Green's theorem [5, Sec. 1.8] and the Maxwell equations, we find the following expression for the absorbed power:

$$
P_{a}=\frac{\omega}{2} \operatorname{Im} \int_{V} d^{3} r k(\mathbf{r})\left[\eta(\mathbf{r})|\mathbf{E}(\mathbf{r})|^{2}+\chi(\mathbf{r})|\mathbf{H}(\mathbf{r})|^{2}\right] .
$$

For a lossy, nonmagnetic $[\chi(\mathbf{r})=0]$ material, $\operatorname{Im}[\eta(\mathbf{r})]$ must be positive in order that the absorbed power be positive. Likewise, for a lossy, nondielectric $[\eta(\mathbf{r})=0]$ material, $\operatorname{Im}[\chi(\mathbf{r})]$ must also be positive.

The Poynting vector associated with the scattered field alone is given by the expression

$$
\mathbf{S}^{(s)}(\mathbf{r})=\frac{c}{8 \pi} \operatorname{Re}\left[\mathbf{E}^{(s)}(\mathbf{r}) \times \mathbf{H}^{(s) *}(\mathbf{r})\right],
$$

from which it follows that the power carried away by the scattered field is given by

$$
P_{s}=\int_{\partial V} d^{2} r \mathbf{S}^{(s)}(\mathbf{r}) \cdot \hat{\mathbf{n}}
$$

Again making use of Green's theorem we obtain for the scattered power the expression

$P_{s}=\frac{-\omega}{2} \operatorname{Im} \int_{V} d^{3} r\left[\mathbf{H}(\mathbf{r}) \cdot \mathbf{H}^{(s)^{*}}(\mathbf{r}) \chi(\mathbf{r})+\mathbf{E}(\mathbf{r}) \cdot \mathbf{E}^{(s)^{*}}(\mathbf{r}) \eta(\mathbf{r})\right]$.

The power supplied to the system is imparted by the incident field. The total power $P_{e}$ extinguished or removed, from the system is due to both scattering and absorption. That is,

$$
P_{e}=P_{a}+P_{s},
$$

where the absorbed power $P_{a}$ and the scattered power $P_{s}$ are given by Eqs. (14) and (17), respectively. Explicitly, the extinguished power is given by the formula

$$
\begin{aligned}
P_{e}= & \frac{c}{2} \operatorname{Im} \int_{V} d^{3} r k(\mathbf{r})\left[\mathbf{E}^{(i)^{*}}(\mathbf{r}) \cdot \mathbf{E}(\mathbf{r}) \eta(\mathbf{r})\right. \\
& \left.+\mathbf{H}^{(i)^{*}}(\mathbf{r}) \cdot \mathbf{H}(\mathbf{r}) \chi(\mathbf{r})\right] .
\end{aligned}
$$

This is our main result for vector fields. As in the case of scalar fields, this result may be understood to relate the total power extinguished from the incident field to the projection of the magnetic and dielectric susceptibilities of the scattering object onto the interference pattern generated by interference of the incident and the total fields within the domain of the scatterer. If the scatterer is in vacuum, then $k(\mathbf{r})=k_{0}$ in the domain of the scatterer and the above equation takes the form

$$
P_{e}=\frac{\omega}{2} \operatorname{Im} \int_{V} d^{3} r\left[\mathbf{E}^{(i)^{*}}(\mathbf{r}) \cdot \mathbf{E}(\mathbf{r}) \eta(\mathbf{r})+\mathbf{H}^{(i) *}(\mathbf{r}) \cdot \mathbf{H}(\mathbf{r}) \chi(\mathbf{r})\right] .
$$

\section{FREE SPACE}

We will now show that when the scatterer is in free space, the general result given in Eq. (20) yields a relationship between the extinguished power and the scattering amplitude of the object. Moreover, our discussion will provide a template for the calculations provided in the subsequent section where we consider the more complicated problem of scattering in a half space.

We suppose that the scattering object is located in the half space $z \geqslant 0$ and the sources of the incident field are located in the half space $z<0$. The most general case, where sources may be located anywhere outside of the object, may be treated by an extension of the analysis presented here. We consider only nonmagnetic materials $(\chi=0)$, though magnetic materials may be included by an analogous approach.

The incident field may be expressed as a superposition of plane waves ([5], Sec. 3.2) with complex vector amplitudes, say, $\mathbf{e}(\mathbf{k})$. The vector $\mathbf{k}=\mathbf{k}_{\|}+\hat{\mathbf{z}} k_{z}, \mathbf{k}_{\|}$being a vector parallel to the plane $z=0, \hat{\mathbf{z}}$ being the unit vector in the positive $z$ direction, and 


$$
k_{z}= \begin{cases}\sqrt{k_{0}^{2}-k_{\|}^{2}} & \text { when } k_{0}^{2}>k_{\|}^{2}, \\ i \sqrt{k_{\|}^{2}-k_{0}^{2}} & \text { when } k_{0}^{2}<k_{\|}^{2} .\end{cases}
$$

The dependence of $k_{z}$ on $k_{\|}$is implied throughout. When the modulus $\left|\mathbf{k}_{\|}\right|$exceeds the free-space wave number $k_{0}$, the quantity $k_{z}$ becomes purely imaginary. Such values of $k_{z}$ correspond to evanescent modes of the incident field that decay exponentially on propagation. The incident electric field is given by the formula

$$
E_{\beta}^{(i)}(\mathbf{r})=\int d^{2} k_{\|} e_{\alpha}(\mathbf{k}) \phi_{\alpha \beta}^{(i)}(\mathbf{r}, \mathbf{k})
$$

Here

$$
\phi_{\alpha \beta}^{(i)}(\mathbf{r}, \mathbf{k})=\left(\delta_{\alpha \beta}-\frac{k_{\alpha} k_{\beta}}{k_{0}^{2}}\right) e^{i \mathbf{k} \cdot \mathbf{r}}
$$

where $\delta_{\alpha \beta}$ is the Kronecker symbol, summation over repeated indices is implied, and the factor $\delta_{\alpha \beta}-k_{\alpha} k_{\beta} / k_{0}^{2}$ ensures transversality of the fields.

Let us introduce the quantity $\phi_{\alpha \beta}(\mathbf{r}, \mathbf{k})$ representing the modes of the the total field associated with the scattering of incident plane waves. That is, the total field produced on scattering of the plane wave $e_{\alpha}(\mathbf{k}) \phi_{\alpha \beta}^{(i)}(\mathbf{r}, \mathbf{k})$ is $e_{\alpha}(\mathbf{k}) \phi_{\alpha \beta}(\mathbf{r}, \mathbf{k})$. Since Eqs. (3) and (4) are linear in the fields, the total electric field is given by the expression

$$
E_{\beta}(\mathbf{r})=\int d^{2} k_{1 \|} e_{\alpha}\left(\mathbf{k}_{1}\right) \phi_{\alpha \beta}\left(\mathbf{k}_{1}, \mathbf{r}\right)
$$

(see Fig. 1). The modes of the total field can be written in terms of incident and scattered field modes:

$$
\phi_{\alpha \beta}(\mathbf{r}, \mathbf{k})=\phi_{\alpha \beta}^{(i)}(\mathbf{r}, \mathbf{k})+\phi_{\alpha \beta}^{(s)}(\mathbf{r}, \mathbf{k})
$$

where $\phi_{\alpha \beta}^{(s)}(\mathbf{r}, \mathbf{k})$ is the scattered field tensor such that the scattered field produced on scattering of a plane wave $e_{\alpha}(\mathbf{k}) \phi_{\alpha \beta}^{(i)}(\mathbf{r}, \mathbf{k})$ is $e_{\alpha}(\mathbf{k}) \phi_{\alpha \beta}^{(s)}(\mathbf{r}, \mathbf{k})$. The scattered field modes $\phi_{\alpha \beta}^{(s)}(\mathbf{r}, \mathbf{k})$ satisfy the integral equation

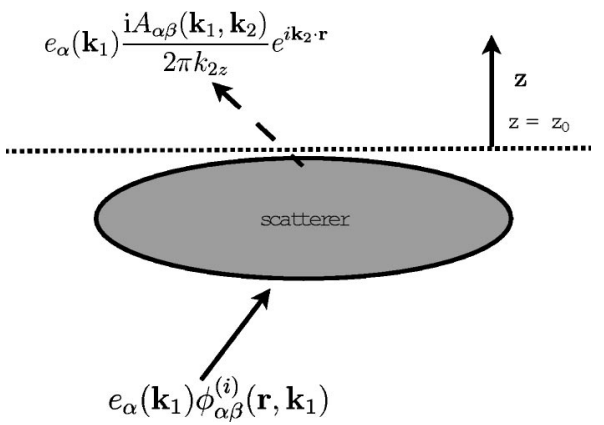

FIG. 1. Illustrating the notation for scattering in free space. An incident wave $e_{\alpha}\left(\mathbf{k}_{1}\right) \phi_{\alpha \beta}^{(i)}\left(\mathbf{r}, \mathbf{k}_{1}\right)$ is represented by the solid line indicating the wave vector $\mathbf{k}_{1}$. A plane wave component of the scattered field with amplitude proportional to $e_{\alpha}\left(\mathbf{k}_{1}\right) A_{\alpha \beta}\left(\mathbf{k}_{1}, \mathbf{k}_{2}\right)$ is represented by a dashed line indicating the wave vector $\mathbf{k}_{2}$.

$$
\phi_{\alpha \beta}^{(s)}(\mathbf{r}, \mathbf{k})=k_{0}^{2} \int d^{3} r^{\prime} G_{\beta \gamma}\left(\mathbf{r}, \mathbf{r}^{\prime}\right) \eta\left(\mathbf{r}^{\prime}\right) \phi_{\alpha \gamma}\left(\mathbf{r}^{\prime}, \mathbf{k}\right) .
$$

In Eq. (26) $G_{\alpha \beta}\left(\mathbf{r}, \mathbf{r}^{\prime}\right)$ is the outgoing Green's tensor that satisfies the equation

$$
-\nabla \times \nabla \times G\left(\mathbf{r}, \mathbf{r}^{\prime}\right)+k_{0}^{2} G\left(\mathbf{r}, \mathbf{r}^{\prime}\right)=-4 \pi I \delta^{(3)}\left(\mathbf{r}-\mathbf{r}^{\prime}\right),
$$

where $\delta^{(3)}\left(\mathbf{r}-\mathbf{r}^{\prime}\right)$ is the three dimensional Dirac delta function and $I$ is the unit tensor. The Green's tensor may be obtained from the Green's function for the scalar case by the formula [6]

$$
G_{\alpha \beta}\left(\mathbf{r}, \mathbf{r}^{\prime}\right)=\left(\delta_{\alpha \beta}+\frac{1}{k_{0}^{2}} \frac{\partial^{2}}{\partial r_{\alpha} \partial r_{\beta}}\right) G_{0}\left(\mathbf{r}, \mathbf{r}^{\prime}\right),
$$

where $G_{0}\left(\mathbf{r}, \mathbf{r}^{\prime}\right)$ is the scalar Green's function for the Helmholtz equation with the following plane wave representation:

$$
G_{0}\left(\mathbf{r}, \mathbf{r}^{\prime}\right)=\frac{i}{2 \pi} \int \frac{d^{2} k_{\|}}{k_{z}} e^{i \mathbf{k}_{\|}\left(\mathbf{r}-\mathbf{r}^{\prime}\right)+i k_{z}\left|z-z^{\prime}\right|} .
$$

We denote reflections through the $z=0$ plane by a tilde. Thus we write $\widetilde{\mathbf{k}}=\mathbf{k}_{\|}-k_{z} \hat{\mathbf{z}}$. Hence, for $z \geqslant z^{\prime}$,

$$
G_{\alpha \beta}\left(\mathbf{r}, \mathbf{r}^{\prime}\right)=\frac{i}{2 \pi} \int \frac{d^{2} k_{\|}}{k_{z}}\left(\delta_{\alpha \beta}-\frac{k_{\alpha} k_{\beta}}{k_{0}^{2}}\right) e^{i \mathbf{k} \cdot\left(\mathbf{r}-\mathbf{r}^{\prime}\right)}
$$

and for $z<z^{\prime}$,

$$
G_{\alpha \beta}\left(\mathbf{r}, \mathbf{r}^{\prime}\right)=\frac{i}{2 \pi} \int \frac{d^{2} k_{\|}}{k_{z}}\left(\delta_{\alpha \beta}-\frac{\tilde{k}_{\alpha} \tilde{k}_{\beta}}{k_{0}^{2}}\right) e^{i \tilde{\mathbf{k}} \cdot\left(\mathbf{r}-\mathbf{r}^{\prime}\right)} .
$$

Thus, $\phi_{\alpha \beta}^{(s)}\left(\mathbf{r}, \mathbf{k}_{1}\right)$ can also be represented in the form of a plane wave decomposition:

$$
\phi_{\alpha \beta}^{(s)}\left(\mathbf{r}, \mathbf{k}_{1}\right)=\frac{i}{2 \pi} \int \frac{d^{2} k_{2 \|}}{k_{2 z}} e^{i \mathbf{k}_{2} \cdot \mathbf{r}} A_{\alpha \beta}\left(\mathbf{k}_{1}, \mathbf{k}_{2}\right),
$$

which defines the scattering amplitude $A_{\alpha \beta}\left(\mathbf{k}_{1}, \mathbf{k}_{2}\right)$. Using the Fourier inversion formula, $A_{\alpha \beta}$ may be seen to be given by the expression

$$
A_{\alpha \beta}\left(\mathbf{k}_{1}, \mathbf{k}_{2}\right)=\frac{k_{2 z}}{2 \pi i} \int_{z=z_{0}} d^{2} r e^{-i \mathbf{k}_{2} \cdot \mathbf{r}} \phi_{\alpha \beta}^{(s)}\left(\mathbf{r}, \mathbf{k}_{1}\right),
$$

where $z=z_{0}>0$ is any plane outside the domain of the scatterer. It can readily be shown that in the far zone of the object $\phi_{\alpha \beta}^{(s)}\left(\mathbf{r}, \mathbf{k}_{1}\right)$ behaves asymptotically as

$$
\phi_{\alpha \beta}^{(s)}\left(\mathbf{r}, \mathbf{k}_{1}\right) \sim \frac{e^{i k_{0} r}}{r} A_{\alpha \beta}\left(\mathbf{k}_{1}, k_{0} \hat{\mathbf{r}}\right) \quad \text { as } k_{0} r \rightarrow \infty
$$

in any fixed direction $\hat{\mathbf{r}}$.

Substituting from Eq. (30) into Eq. (26) we obtain for $\phi_{\alpha \beta}^{(s)}(\mathbf{r}, \mathbf{k})$ the expression 


$$
\begin{aligned}
\phi_{\alpha \beta}^{(s)}(\mathbf{r}, \mathbf{k})= & k_{0}^{2} \frac{i}{2 \pi} \int d^{3} r^{\prime} \int \frac{d^{2} k_{\|}}{k_{z}} \\
& \times\left(\delta_{\beta \gamma}-\frac{k_{\beta} k_{\gamma}}{k_{0}^{2}}\right) e^{i \mathbf{k} \cdot\left(\mathbf{r}-\mathbf{r}^{\prime}\right)} \eta\left(\mathbf{r}^{\prime}\right) \phi_{\alpha \gamma}\left(\mathbf{r}^{\prime}, \mathbf{k}\right),
\end{aligned}
$$

where we again assume that $z \geqslant z_{0}>0$. Substituting this expression for $\phi_{\alpha \beta}^{(s)}(\mathbf{r}, \mathbf{k})$ into Eq. (33), we obtain the following expression for the scattering amplitude in terms of the outgoing wave vector $\mathbf{k}_{2}$ :

$$
\begin{aligned}
A_{\alpha \beta}\left(\mathbf{k}_{1}, \mathbf{k}_{2}\right)= & k_{0}^{2} \int_{V} d^{3} r^{\prime}\left(\delta_{\beta \gamma}-\frac{k_{2 \beta} k_{2 \gamma}}{k_{0}^{2}}\right) \\
& \times e^{-i \mathbf{k}_{2} \cdot \mathbf{r}^{\prime}} \eta\left(\mathbf{r}^{\prime}\right) \phi_{\alpha \gamma}\left(\mathbf{r}^{\prime}, \mathbf{k}_{1}\right) .
\end{aligned}
$$

Using our main formula (20) for the extinguished power and assuming that $\chi(\mathbf{r})=0$, we see that the extinguished power is given by the expression

$$
\begin{aligned}
P_{e}= & \frac{\omega}{2} \operatorname{Im} \int d^{2} k_{1 \|} \int d^{2} k_{2 \|} \int_{V} d^{3} r e_{\alpha}\left(\mathbf{k}_{1}\right) \phi_{\alpha \gamma}\left(\mathbf{r}, \mathbf{k}_{1}\right) \\
& \times \eta(\mathbf{r}) e_{\beta}^{*}\left(\mathbf{k}_{2}\right) \phi_{\beta \gamma}^{(i) *}\left(\mathbf{r}, \mathbf{k}_{2}\right) .
\end{aligned}
$$

Thus Eqs. (23), (36), and (37) imply that

$$
P_{e}=\frac{\omega}{2} \operatorname{Im} \int d^{2} k_{1 \|} \int d^{2} k_{2 \|} e_{\beta}^{*}\left(\mathbf{k}_{2}\right) e_{\alpha}\left(\mathbf{k}_{1}\right) A_{\alpha \beta}\left(\mathbf{k}_{1}, \mathbf{k}_{2}^{*}\right) .
$$

The case when a single plane wave is incident on the scatterer may be recovered by setting $\mathbf{e}(\mathbf{k})=\mathbf{e}_{0} \delta^{(2)}\left(\mathbf{k}_{\|}-\mathbf{k}_{0 \|}\right)$. By defining the cross section as $\sigma_{e}=P_{e} /\left|\mathbf{e}_{0}\right|^{2}$, we obtain Eq. (1).

The scattering amplitude may be expressed more compactly. Due to the presence of the projection onto transverse modes, $\delta_{\alpha \beta}-k_{\alpha} k_{\beta} / k_{0}^{2}$ in Eq. (36), the scattering amplitude has a nontrivial null space corresponding to nontransverse field vectors. It is thus appropriate to express the complex vector field amplitudes in a basis such as the transverse electric (TE) and transverse magnetic (TM) field vectors. Suppose $\mathbf{p}_{1}(\mathbf{k})$ and $\mathbf{p}_{2}(\mathbf{k})$ are two orthonormal eigenvectors of the projection onto the transverse fields $\delta_{\alpha \beta}-k_{\alpha} k_{\beta} / k_{0}^{2}$. Defining $\mathcal{A}_{i j}\left(\mathbf{k}_{1}, \mathbf{k}_{2}^{*}\right)=p_{j \beta}\left(\mathbf{k}_{1}\right) p_{i \alpha}^{*}\left(\mathbf{k}_{2}\right) A_{\alpha \beta}\left(\mathbf{k}_{1}, \mathbf{k}_{2}^{*}\right)$ and $a_{j}=\mathbf{e} \cdot \mathbf{p}_{j}$, we obtain

$$
P_{e}=\frac{\omega}{2} \operatorname{Im} \int d^{2} k_{1 \|} \int d^{2} k_{2 \|} a_{j}^{*}\left(\mathbf{k}_{2}\right) a_{i}\left(\mathbf{k}_{1}\right) \mathcal{A}_{i j}\left(\mathbf{k}_{1}, \mathbf{k}_{2}^{*}\right) .
$$

This result is analogous to that obtained for scalar fields [4] except that in the vector formulation, the extinguished power depends on the complex vector amplitude of the incident field. It may be seen that the power extinguished from the incident field is removed by interference of the scattered field with the incident field. Equation (39) expresses this relationship in terms of the plane wave components of the incident field and takes into account the cross terms between these modes.

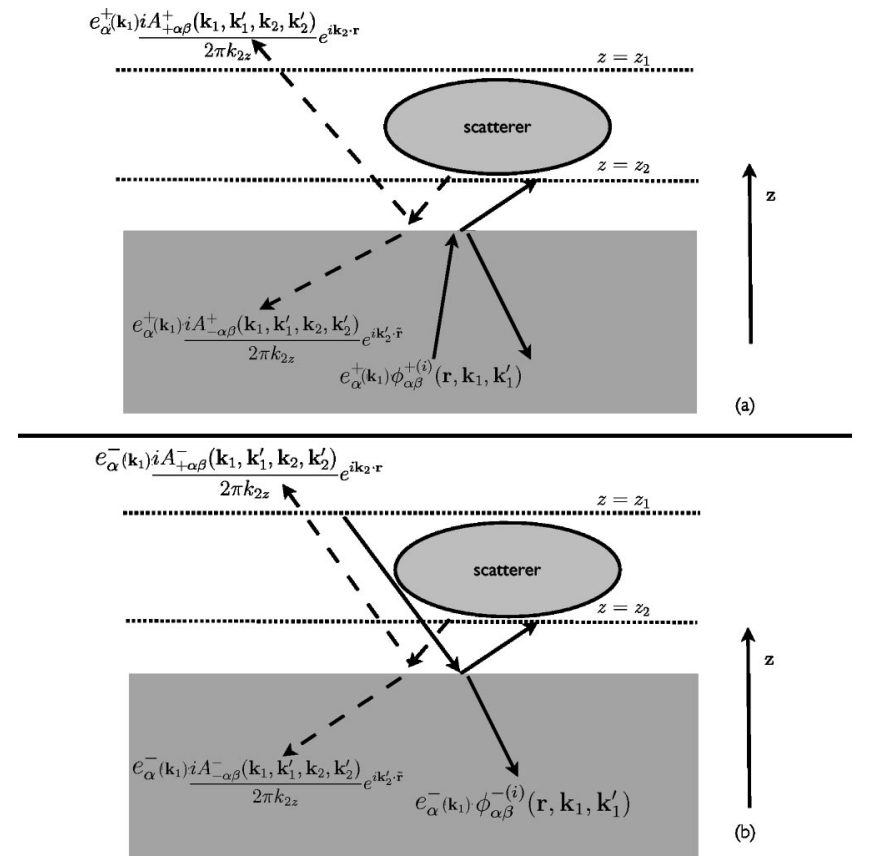

FIG. 2. Illustrating the notation for the half-space problem. In (a), an incident wave $e_{\alpha}^{+}\left(\mathbf{k}_{1}\right) \phi_{\alpha \beta}^{+(i)}\left(\mathbf{r}, \mathbf{k}_{1}, \mathbf{k}_{1}^{\prime}\right)$ associated with sources in the lower half space is represented by a solid line indicating the wave vector of the three plane wave components of the field. The wave vectors of the plane wave components of a mode of the scattered field are represented by a dashed line. The two codirectional plane wave components of the scattered fieldin the upper half space combine to produce an outgoing plane wave with wave vector $\mathbf{k}_{2}$ and amplitude proportional to $e_{\alpha}^{+}\left(\mathbf{k}_{1}\right) A_{+\alpha \beta}^{+}\left(\mathbf{k}_{1}, \mathbf{k}_{1}^{\prime}, \mathbf{k}_{2}, \mathbf{k}_{2}^{\prime}\right)$. The plane wave component of the scattered mode in the lower half space is proportional to $e_{\alpha}^{+}\left(\mathbf{k}_{1}\right) A_{-\alpha \beta}^{+}\left(\mathbf{k}_{1}, \mathbf{k}_{1}^{\prime}, \mathbf{k}_{2}, \mathbf{k}_{2}^{\prime}\right)$. In (b), the notation is similarly illustrated with a different mode of the incident field $e_{\alpha}^{-}\left(\mathbf{k}_{1}\right) \phi_{\alpha \beta}^{-(i)}\left(\mathbf{r}, \mathbf{k}_{1}, \mathbf{k}_{1}^{\prime}\right)$ generated by sources in the upper half space.

\section{HALF SPACE}

We now consider the problem of scattering from an object in the presence of a planar interface separating two semiinfinite half spaces. It is assumed that the scatterer is of finite size and is located in the region $z_{1} \geqslant z \geqslant z_{2}$ as shown in Fig. 2 . The half space $z>0$ is assumed to be vacuum. The other half space $z<0$ is assumed to be occupied by a material whose index of refraction is $n$. The field obeys Eqs. (9) and (10) with

$$
k(\mathbf{r})= \begin{cases}k_{0} & \text { for } z \geqslant 0, \\ n k_{0} & \text { for } z<0 .\end{cases}
$$

In the domain of the scatterer, the incident field obeys Eq. (9) and can be represented as a superposition of plane wave modes. We exclude the case where sources are located in the region of the scatterer. The modes of the incident field may be seen to consist of three plane wave components, usually called the incoming, reflected, and transmitted parts of the field (see Fig. 2) with coefficients obtained by imposing the boundary conditions

$$
\hat{\mathbf{z}} \times\left.\mathbf{E}(\mathbf{r})\right|_{z=0^{+}}=\hat{\mathbf{z}} \times\left.\mathbf{E}(\mathbf{r})\right|_{z=0^{-}},
$$




$$
\hat{\mathbf{z}} \times \boldsymbol{\nabla} \times\left.\mathbf{E}(\mathbf{r})\right|_{z=0^{+}}=\hat{\mathbf{z}} \times \boldsymbol{\nabla} \times\left.\mathbf{E}(\mathbf{r})\right|_{z=0^{-}} .
$$

The modes of the incident field generated by sources in the lower half space may thus be seen to be given by the expression

$$
\begin{aligned}
\phi_{\alpha \beta}^{+(i)}\left(\mathbf{r}, \mathbf{k}, \mathbf{k}^{\prime}\right)= & \theta(-z)\left[\left(\delta_{\alpha \beta}-\frac{k_{\alpha}^{\prime} k_{\beta}^{\prime}}{n^{2} k_{0}^{2}}\right) e^{i \mathbf{k}^{\prime} \cdot \mathbf{r}}+R_{\alpha \beta}^{\prime}\left(\mathbf{k}, \mathbf{k}^{\prime}\right) e^{i \mathbf{k}^{\prime} \cdot \mathbf{r}}\right] \\
& +\theta(z) T_{\alpha \beta}^{\prime}\left(\mathbf{k}, \mathbf{k}^{\prime}\right) e^{i \mathbf{k} \cdot \mathbf{r}} .
\end{aligned}
$$

The modes of the field generated by sources in the upper half space are given by the expression

$$
\begin{aligned}
\phi_{\alpha \beta}^{-(i)}\left(\mathbf{r}, \mathbf{k}, \mathbf{k}^{\prime}\right)= & \theta(z)\left[\left(\delta_{\alpha \beta}-\frac{\tilde{k}_{\alpha} \tilde{k}_{\beta}}{k_{0}^{2}}\right) e^{i \mathbf{k} \cdot \mathbf{r}}+R_{\alpha \beta}\left(\mathbf{k}, \mathbf{k}^{\prime}\right) e^{i \mathbf{k} \cdot \mathbf{r}}\right] \\
& +\theta(-z) T_{\alpha \beta}\left(\mathbf{k}, \mathbf{k}^{\prime}\right) e^{i \tilde{\mathbf{k}}^{\prime} \cdot \mathbf{r}},
\end{aligned}
$$

where $R\left(\mathbf{k}, \mathbf{k}^{\prime}\right), R^{\prime}\left(\mathbf{k}, \mathbf{k}^{\prime}\right), T\left(\mathbf{k}, \mathbf{k}^{\prime}\right)$, and $T^{\prime}\left(\mathbf{k}, \mathbf{k}^{\prime}\right)$ are the reflection and transmission tensors that are discussed in the Appendix and $\theta(z)$ is the Heaviside step function. In the upper half space, the wave vectors $\mathbf{k}$ are given by Eq. (45) and in the lower half space the wave vectors $\mathbf{k}^{\prime}=\mathbf{k}_{\|}+k_{z}^{\prime} \hat{\mathbf{z}}$ and $\widetilde{\mathbf{k}}^{\prime}=\mathbf{k}_{\|}-k_{z}^{\prime} \hat{\mathbf{z}}$ where

$$
k_{z}^{\prime}=\left\{\begin{array}{l}
\sqrt{n^{2} k_{0}^{2}-k_{\|}^{2}} \quad \text { when } k_{0}^{2}>k_{\|}^{2}, \\
i \sqrt{k_{\|}^{2}-n^{2} k_{0}^{2}} \quad \text { when } n^{2} k_{0}^{2}<k_{\|}^{2} .
\end{array}\right.
$$

It can be verified that these modes are orthogonal and span the space of incident fields satisfying Eq. (7). The incident field can therefore be written in the form

$$
E_{\beta}^{(i)}(\mathbf{r})=\int d^{2} k_{\|}\left[e_{\alpha}^{+}(\mathbf{k}) \phi_{\alpha \beta}^{+(i)}\left(\mathbf{r}, \mathbf{k}, \mathbf{k}^{\prime}\right)+e_{\alpha}^{-}(\mathbf{k}) \phi_{\alpha \beta}^{-(i)}\left(\mathbf{r}, \mathbf{k}, \mathbf{k}^{\prime}\right)\right] .
$$

We will use the more compact notation,

$$
E_{\beta}^{(i)}(\mathbf{r})=\sum_{n= \pm} \int d^{2} k_{\|} e_{\alpha}^{n}(\mathbf{k}) \phi_{\alpha \beta}^{n(i)}\left(\mathbf{r}, \mathbf{k}, \mathbf{k}^{\prime}\right) .
$$

The Green's tensor $G_{\alpha \beta}\left(\mathbf{r}, \mathbf{r}^{\prime}\right)$ for this case satisfies the equation

$$
-\boldsymbol{\nabla} \times \boldsymbol{\nabla} \times G\left(\mathbf{r}, \mathbf{r}^{\prime}\right)+n^{2}(z) k_{0}^{2} G\left(\mathbf{r}, \mathbf{r}^{\prime}\right)=-4 \pi I \delta^{(3)}\left(\mathbf{r}-\mathbf{r}^{\prime}\right),
$$

and obeys the boundary conditions given in Eqs. (41) and (42). The Green's tensor may be expressed as a superposition of outgoing modes satisfying the above boundary conditions. For $z^{\prime} \geqslant 0$, the only case of interest here, it is given by [7]

$$
\begin{aligned}
G_{\alpha \beta}\left(\mathbf{r}, \mathbf{r}^{\prime}\right)= & \frac{i}{2 \pi} \int \frac{d^{2} k_{\|}}{k_{z}} e^{i \mathbf{k}_{\|} \cdot\left(\mathbf{r}-\mathbf{r}^{\prime}\right)}\left\{\theta(-z) T_{\alpha \beta}\left(\mathbf{k}, \mathbf{k}^{\prime}\right) e^{i\left(k_{z} z^{\prime}-k_{z}^{\prime} z\right)}+\theta(z)\left[R_{\alpha \beta}\left(\mathbf{k}, \mathbf{k}^{\prime}\right) e^{i k_{z}\left(z+z^{\prime}\right)}+\theta\left(z-z^{\prime}\right)\left(\delta_{\alpha \beta}-\frac{k_{\alpha} k_{\beta}}{k_{0}^{2}}\right) e^{i k_{z}\left(z-z^{\prime}\right)}\right.\right. \\
& \left.\left.+\theta\left(z^{\prime}-z\right)\left(\delta_{\alpha \beta}-\frac{\tilde{k}_{\alpha} \tilde{k}_{\beta}}{k_{0}^{2}}\right) e^{i k_{z}\left(z^{\prime}-z\right)}\right]\right\} .
\end{aligned}
$$

It is again useful to introduce tensor modes of the total field $\phi_{\alpha \beta}^{ \pm}\left(\mathbf{r}, \mathbf{k}, \mathbf{k}^{\prime}\right)$. The total field modes are superpositions of the incident and scattered field modes given by the formula

$$
\phi_{\alpha \beta}^{ \pm}\left(\mathbf{r}, \mathbf{k}, \mathbf{k}^{\prime}\right)=\phi_{\alpha \beta}^{ \pm(i)}\left(\mathbf{r}, \mathbf{k}, \mathbf{k}^{\prime}\right)+\phi_{\alpha \beta}^{ \pm(s)}\left(\mathbf{r}, \mathbf{k}, \mathbf{k}^{\prime}\right),
$$

where the scattered field modes satisfy the integral equation

$$
\phi_{\alpha \beta}^{ \pm(s)}\left(\mathbf{r}, \mathbf{k}, \mathbf{k}^{\prime}\right)=k_{0}^{2} \int d^{3} r^{\prime} G_{\beta \gamma}\left(\mathbf{r}, \mathbf{r}^{\prime}\right) \eta\left(\mathbf{r}^{\prime}\right) \phi_{\alpha \gamma}^{ \pm}\left(\mathbf{r}^{\prime}, \mathbf{k}_{1}, \mathbf{k}_{1}^{\prime}\right)
$$

The total field vector can then be expressed as a sum of total field modes,

$$
E_{\beta}(\mathbf{r})=\sum_{n= \pm} \int d^{2} k_{\|} e_{\alpha}^{n}(\mathbf{k}) \phi_{\alpha \beta}^{n}\left(\mathbf{r}, \mathbf{k}, \mathbf{k}^{\prime}\right)
$$

and the scattered field vector can be written as

$$
E_{\beta}^{(s)}(\mathbf{r})=\sum_{n= \pm} \int d^{2} k_{\|} e_{\alpha}^{n}(\mathbf{k}) \phi_{\alpha \beta}^{n(s)}\left(\mathbf{r}, \mathbf{k}, \mathbf{k}^{\prime}\right) .
$$

Substituting into Eq. (19) the expressions for the fields given in Eqs. (52) and (53) we obtain the formula

$$
\begin{aligned}
P_{e}= & \frac{\omega}{2} \operatorname{Im} \int d^{3} r \int d^{2} k_{1 \|} \int d^{2} k_{2 \|} \sum_{m, n= \pm} e_{\alpha}^{m^{*}}\left(\mathbf{k}_{2}\right) e_{\beta}^{n}\left(\mathbf{k}_{1}\right) \\
& \times \eta(\mathbf{r}) \phi_{\alpha \delta}^{m(i)^{*}}\left(\mathbf{r}, \mathbf{k}_{2}, \mathbf{k}_{2}^{\prime}\right) \phi_{\beta \delta}^{m}\left(\mathbf{r}, \mathbf{k}_{1}, \mathbf{k}_{1}^{\prime}\right)
\end{aligned}
$$

The modes of the scattered field may be expressed as a superposition of plane waves weighted by the scattering amplitude. In the upper half space the modes of the scattered field are given by the formula

$$
\phi_{\alpha \beta}^{ \pm(s)}\left(\mathbf{r}, \mathbf{k}_{1}, \mathbf{k}_{1}^{\prime}\right)=\frac{i}{2 \pi} \int \frac{d^{2} k_{2 \|}}{k_{2 z}} A_{+\alpha \beta}^{ \pm}\left(\mathbf{k}_{1}, \mathbf{k}_{1}^{\prime}, \mathbf{k}_{2}, \mathbf{k}_{2}^{\prime}\right) e^{i \mathbf{k}_{2} \cdot \mathbf{r}},
$$

and the modes of the scattered field in the lower half space are given by the expression, 


$$
\phi_{\alpha \beta}^{ \pm(s)}\left(\mathbf{r}, \mathbf{k}_{1}, \mathbf{k}_{1}^{\prime}\right)=\frac{i}{2 \pi} \int \frac{d^{2} k_{2 \|}}{k_{2 z}^{\prime}} A_{-\alpha \beta}^{ \pm}\left(\mathbf{k}_{1}, \mathbf{k}_{1}^{\prime}, \mathbf{k}_{2}, \mathbf{k}_{2}^{\prime}\right) e^{i \hat{\mathbf{k}}_{2}^{\prime} \cdot \mathbf{r}} .
$$

The asymptotic form of the scattered field is given by expressions analogous to Eq. (34). From these expressions it may readily be seen that

$$
\begin{aligned}
A_{+\alpha \beta}^{ \pm}\left(\mathbf{k}_{1}, \mathbf{k}_{1}^{\prime}, \mathbf{k}_{2}, \mathbf{k}_{2}^{\prime}\right)= & \frac{-i k_{2 z} e^{-i k_{2 z} z_{1}}}{2 \pi} \int_{z=z_{1}} d^{2} r e^{-i \mathbf{k}_{2 \|} \mathbf{r}} \\
& \times \phi_{\alpha \beta}^{ \pm(s)}\left(\mathbf{r}, \mathbf{k}_{1}, \mathbf{k}_{1}^{\prime}\right), \\
A_{-\alpha \beta}^{ \pm}\left(\mathbf{k}_{1}, \mathbf{k}_{1}^{\prime}, \mathbf{k}_{2}, \mathbf{k}_{2}^{\prime}\right)= & \frac{-i k_{2 z}^{\prime} e^{-i k_{2 z}^{\prime} z_{2}}}{2 \pi} \int_{z=z_{2}} d^{2} r e^{-i \mathbf{k}_{2 \|}^{\prime} \mathbf{r}} \\
& \times \phi_{\alpha \beta}^{ \pm(s)}\left(\mathbf{r}, \mathbf{k}_{1}, \mathbf{k}_{1}^{\prime}\right) .
\end{aligned}
$$

Making use of the explicit form for the Green's tensor and the above results for the scattering amplitude and Eq. (51), we obtain the following expressions for the scattering amplitude:

$$
\begin{aligned}
A_{+\alpha \beta}^{ \pm}\left(\mathbf{k}_{1}, \mathbf{k}_{1}^{\prime}, \mathbf{k}_{2}, \mathbf{k}_{2}^{\prime}\right)= & k_{0}^{2} \int d^{3} r\left[\left(\delta_{\beta \gamma}-\frac{k_{2 \beta} k_{2 \gamma}}{k_{0}^{2}}\right) e^{-i \mathbf{k}_{2} \cdot \mathbf{r}}\right. \\
& \left.+R_{\beta \gamma}\left(\mathbf{k}_{2}, \mathbf{k}_{2}^{\prime}\right) e^{-i \tilde{\mathbf{k}}_{2} \cdot \mathbf{r}}\right] \\
& \times \eta(\mathbf{r}) \phi_{\alpha \gamma}^{ \pm}\left(\mathbf{r}, \mathbf{k}_{1}, \mathbf{k}_{1}^{\prime}\right), \\
A_{-\alpha \beta}^{ \pm}\left(\mathbf{k}_{1}, \mathbf{k}_{1}^{\prime}, \mathbf{k}_{2}, \mathbf{k}_{2}^{\prime}\right)= & \frac{k_{0}^{2} k_{2 z}^{\prime}}{k_{2 z}} \int d^{3} r T_{\beta \gamma}\left(\mathbf{k}_{2}, \mathbf{k}_{2}^{\prime}\right) e^{-\tilde{i}_{2} \cdot \mathbf{r}} \\
& \times \eta(\mathbf{r}) \phi_{\alpha \gamma}^{ \pm}\left(\mathbf{r}, \mathbf{k}_{1}, \mathbf{k}_{1}^{\prime}\right) .
\end{aligned}
$$

It is useful to note that, with the aid of several identities given in the Appendix, the field modes in the domain of the scatterer, $z^{\prime} \geqslant 0$, given in Eqs (50) and (51), can be written as

$$
\begin{aligned}
\phi_{\alpha \delta}^{+(i)^{*}}\left(\mathbf{r}, \mathbf{k}, \mathbf{k}^{\prime}\right)= & T_{\mu \alpha}^{\prime}\left(\mathbf{k}^{*}, \mathbf{k}^{\prime *}\right)\left[\left(\delta_{\mu \delta}-\frac{k_{\mu}^{*} k_{\delta}^{*}}{k_{0}^{2}}\right) e^{-i \mathbf{k}^{*} \cdot \mathbf{r}}\right. \\
& \left.+R_{\mu \delta}\left(\mathbf{k}^{*}, \mathbf{k}^{\prime *}\right) e^{-i \mathbf{k}^{*} \cdot \mathbf{r}}\right] \\
& +\frac{k_{z}^{\prime}}{k_{z}} R_{\mu \alpha}^{\prime}\left(\mathbf{k}^{*}, \mathbf{k}^{\prime *}\right) T_{\mu \delta}\left(\mathbf{k}^{*}, \mathbf{k}^{\prime *}\right) e^{-i \tilde{\mathbf{k}}^{*} \cdot \mathbf{r}},
\end{aligned}
$$

$$
\begin{aligned}
\phi_{\alpha \delta}^{-(i) *}\left(\mathbf{r}, \mathbf{k}, \mathbf{k}^{\prime}\right)= & R_{\mu \alpha}\left(\mathbf{k}^{*}, \mathbf{k}^{\prime *}\right)\left[\left(\delta_{\mu \delta}-\frac{k_{\mu}^{*} k_{\delta}^{*}}{k_{0}^{2}}\right) e^{-i \mathbf{k}^{*} \cdot \mathbf{r}}\right. \\
& \left.+R_{\mu \delta}\left(\mathbf{k}^{*}, \mathbf{k}^{\prime *}\right) e^{-i \mathbf{k}^{*} \cdot \mathbf{r}}\right] \\
& +\frac{k_{z}^{\prime}}{k_{z}} T_{\mu \alpha}\left(\mathbf{k}^{*}, \mathbf{k}^{\prime *}\right) T_{\mu \delta}\left(\mathbf{k}^{*}, \mathbf{k}^{\prime *}\right) e^{-i \mathbf{k}^{*} \cdot \mathbf{r}} .
\end{aligned}
$$

Substituting these expressions into Eq. (54) and comparing the results with Eqs. (59) and (60), we find that for a field incident from the lower half space $e_{\gamma}^{+}(\mathbf{k}) \phi_{\gamma \beta}^{+(i)}\left(\mathbf{r}, \mathbf{k}, \mathbf{k}^{\prime}\right)$ the extinguished power may be expressed as

$$
\begin{aligned}
P_{e}= & \frac{\omega}{2} \operatorname{Im}\left\{e _ { \alpha } ^ { + * } ( \mathbf { k } ) e _ { \beta } ^ { + } ( \mathbf { k } ) \left[T_{\gamma \alpha}^{\prime *}\left(\mathbf{k}, \mathbf{k}^{\prime}\right) A_{+\gamma \beta}^{+}\left(\mathbf{k}, \mathbf{k}^{\prime}, \mathbf{k}^{*}, \mathbf{k}^{\prime *}\right)\right.\right. \\
& \left.\left.+R_{\gamma \alpha}^{\prime *}\left(\mathbf{k}, \mathbf{k}^{\prime}\right) A_{-\gamma \beta}^{+}\left(\mathbf{k}, \mathbf{k}^{\prime}, \mathbf{k}^{*}, \mathbf{k}^{\prime *}\right)\right]\right\} .
\end{aligned}
$$

This result has a clear physical interpretation. In the absence of the scatterer, each component of the incident field imparts a certain amount of power to the far zone via the outgoing plane waves reflected from and transmitted through the planar boundary $(z=0)$ of the half spaces. The scatterer depletes, or extinguishes, some power from the incident field. In order to properly account for the total power, the field produced upon scattering must interfere coherently with the incident field to extinguish incident power. Thus the incident field modes $\phi_{\gamma \beta}^{+(i)}\left(\mathbf{r} ; \mathbf{k}_{1}, \mathbf{k}_{1}^{\prime}\right)$ deliver power to the far zone through the plane waves $e^{i \tilde{\mathbf{k}}_{1}^{\prime} \cdot \mathbf{r}}$ and $e^{i \mathbf{k}_{1} \cdot \mathbf{r}}$, and the extinguished power is directly related to the amplitude of the scattered plane waves in those same directions. Likewise, the power extinguished from the field $e_{\gamma}^{-} \phi_{\gamma \beta}^{-(i)}$ is given by

$$
\begin{aligned}
P_{e}= & \frac{\omega}{2} \operatorname{Im}\left\{e _ { \alpha } ^ { - * } ( \mathbf { k } ) e _ { \beta } ^ { - } ( \mathbf { k } ) \left[R_{\gamma \alpha}^{*}\left(\mathbf{k}, \mathbf{k}^{\prime}\right) A_{+\gamma \beta}^{-}\left(\mathbf{k}, \mathbf{k}^{\prime}, \mathbf{k}^{*}, \mathbf{k}^{\prime *}\right)\right.\right. \\
& \left.\left.+T_{\gamma \alpha}^{*}\left(\mathbf{k}, \mathbf{k}^{\prime}\right) A_{-\gamma \beta}^{-}\left(\mathbf{k}, \mathbf{k}^{\prime}, \mathbf{k}^{*}, \mathbf{k}^{\prime *}\right)\right]\right\} .
\end{aligned}
$$

When the incident field is not a single incident mode, but consists of a superposition of modes, we find that the extinguished power is given by the formula

$$
\begin{aligned}
P_{e}= & \frac{\omega}{2} \sum_{n= \pm} \operatorname{Im}\left\{\int d^{2} k_{1 \|} d^{2} k_{2 \|} e_{\alpha}^{+*}\left(\mathbf{k}_{2}\right) e_{\beta}^{n}\left(\mathbf{k}_{1}\right)\right. \\
& \times\left[T_{\gamma \alpha}^{\prime *}\left(\mathbf{k}_{2}, \mathbf{k}_{2}^{\prime}\right) A_{+\gamma \beta}^{n}\left(\mathbf{k}_{1}, \mathbf{k}_{1}^{\prime}, \mathbf{k}_{2}^{*}, \mathbf{k}_{2}^{\prime *}\right)\right. \\
& \left.+R_{\gamma \alpha}^{\prime *}\left(\mathbf{k}_{2}, \mathbf{k}_{2}^{\prime}\right) A_{-\gamma \beta}^{n}\left(\mathbf{k}_{1}, \mathbf{k}_{1}^{\prime}, \mathbf{k}_{2}^{*}, \mathbf{k}_{2}^{\prime *}\right)\right] \\
& +\int d^{2} k_{1 \|} d^{2} k_{2 \|} e_{\beta}^{n}\left(\mathbf{k}_{1}\right) e_{\alpha}^{-*}\left(\mathbf{k}_{2}\right) \\
& \times\left[T_{\gamma \alpha}^{*}\left(\mathbf{k}_{2}, \mathbf{k}_{2}^{\prime}\right) A_{-\gamma \beta}^{n}\left(\mathbf{k}_{1}, \mathbf{k}_{1}^{\prime}, \mathbf{k}_{2}^{*}, \mathbf{k}_{2}^{\prime *}\right)\right. \\
& \left.\left.+R_{\gamma \alpha}^{*}\left(\mathbf{k}_{2}, \mathbf{k}_{2}^{\prime}\right) A_{+\gamma \beta}^{n}\left(\mathbf{k}_{1}, \mathbf{k}_{1}, \mathbf{k}_{2}^{\prime *}, \mathbf{k}_{2}^{\prime *}\right)\right]\right\} .
\end{aligned}
$$

\section{DISCUSSION}

The results presented here lend insight into the processes by which energy conservation is manifest in the scattering of electromagnetic fields. A number of applications are suggested. It is interesting to note that in the first Born approximation, when the scatterer is in free space, the extinguished power may be seen to be the projection of the incident intensity on the imaginary part of the susceptibility, 


$$
P_{e}=\frac{\omega}{2} \int_{V} d^{3} r \mathbf{E}^{(i)}(\mathbf{r}) \cdot \mathbf{E}^{(i)^{*}}(\mathbf{r}) \operatorname{Im}\left[\eta(\mathbf{r})+O\left(\eta^{2}\right)\right] .
$$

This formula suggests a manner in which object structure may be investigated. If the intensity of the incident field forms the kernel of an invertible transformation, then the object structure as described by $\operatorname{Im}[\eta(\mathbf{r})]$ may be found from power extinction measurements. In Refs. [8,9] the incident field was taken to consist of two plane waves and consequently the extinguished power was found to be related to a Fourier transform [8] or to a Fourier-Laplace transform [9] of the object. However the analyses presented in [8] and [9] were carried out for a scalar field. The present results provide a framework in which to extend those works to electromagnetic fields for cases where polarization effects may be important.

The results of this paper may be readily extended to partially coherent fields. The quadratic products of field amplitudes appearing in Eq. (65) must then be replaced by second order coherence tensors. The results may then be applied to problems such as the scattering of partially coherent electromagnetic beams.

\section{ACKNOWLEDGMENTS}

This research was supported by the U.S. Air Force Office of Scientific Research under Contract No. F49620-03-10138, by the Engineering Research Program at the U.S. Department of Energy under Grant No. DE-FG02-ER 45992, and by the Air Force Research Laboratory (AFRC) under Contract No. FA 9451-04-C-0296. P.S.C. would like to acknowledge support by the National Science Foundation under CAREER Grant No. 0239265 and U.S. Air Force MURI Grant No. F49620-03-1-0379. J.S. acknowledges support from the NSF under Grant No. DMR 0425780. We would also like to thank Hugo Schouten for many useful discussions.

\section{APPENDIX}

The reflection and transmission coefficients in the upper and lower half spaces may be obtained by first projecting the field onto the TE-TM basis, then multiplying by the appropriate Fresnel coefficients and projecting back onto the original basis. We denote by $R$ and $R^{\prime}$ reflection in the upper and lower half spaces, respectively, and by $T$ and $T^{\prime}$ transmission from the upper half space into the lower half space and transmission from the lower half space into the upper half space, respectively. $P$ and $P^{\prime}$ are projection operators onto the TE-TM basis in the upper and lower half spaces, respectively, and are discussed below. We find for the reflection and transmission tensors the expressions

$$
\begin{gathered}
R_{\alpha \beta}\left(\mathbf{k}, \mathbf{k}^{\prime}\right)=P_{\gamma \alpha}(\mathbf{k}) r_{\gamma \delta}\left(\mathbf{k}, \mathbf{k}^{\prime}\right) P_{\delta \beta}(\widetilde{\mathbf{k}}), \\
R_{\alpha \beta}^{\prime}\left(\mathbf{k}, \mathbf{k}^{\prime}\right)=P_{\gamma \alpha}^{\prime}\left(\tilde{\mathbf{k}}^{\prime}\right) r_{\gamma \delta}^{\prime}\left(\mathbf{k}, \mathbf{k}^{\prime}\right) P_{\delta \beta}^{\prime}\left(\mathbf{k}^{\prime}\right),
\end{gathered}
$$

$$
\begin{aligned}
& T_{\alpha \beta}\left(\mathbf{k}, \mathbf{k}^{\prime}\right)=P_{\gamma \alpha}^{\prime}\left(\widetilde{\mathbf{k}}^{\prime}\right) t_{\gamma \delta}\left(\mathbf{k}, \mathbf{k}^{\prime}\right) P_{\delta \beta}(\widetilde{\mathbf{k}}), \\
& T_{\alpha \beta}^{\prime}\left(\mathbf{k}, \mathbf{k}^{\prime}\right)=P_{\gamma \alpha}(\mathbf{k}) t_{\gamma \delta}^{\prime}\left(\mathbf{k}, \mathbf{k}^{\prime}\right) P_{\delta \beta}^{\prime}\left(\mathbf{k}^{\prime}\right),
\end{aligned}
$$

where $r$ and $t$ are given by

$$
r=\left(\begin{array}{cc}
\frac{n^{2} k_{z}-k_{z}^{\prime}}{n^{2} k_{z}+k_{z}^{\prime}} & 0 \\
0 & \frac{k_{z}-k_{z}^{\prime}}{k_{z}+k_{z}^{\prime}}
\end{array}\right)
$$

and

$$
t=\left(\begin{array}{cc}
\frac{2 n k_{z}}{n^{2} k_{z}+k_{z}^{\prime}} & 0 \\
0 & \frac{2 k_{z}}{k_{z}+k_{z}^{\prime}}
\end{array}\right),
$$

and $r^{\prime}=-r, t^{\prime}=k_{z}^{\prime} t / k_{z}$. The projection operator onto the TE-TM basis in the upper half space is given by

$$
P(\mathbf{k})=\frac{1}{\sqrt{k_{x}^{2}+k_{y}^{2}} k_{0}}\left(\begin{array}{ccc}
-k_{x} k_{z} & -k_{y} k_{z} & k_{x}^{2}+k_{y}^{2} \\
-k_{y} k_{0} & k_{x} k_{0} & 0
\end{array}\right)
$$

and in the lower half space by

$$
P^{\prime}\left(\mathbf{k}^{\prime}\right)=\frac{1}{\sqrt{k_{x}^{2}+k_{y}^{2}} n k_{0}}\left(\begin{array}{ccc}
-k_{x} k_{z}^{\prime} & -k_{y} k_{z}^{\prime} & k_{x}^{2}+k_{y}^{2} \\
-k_{y} n k_{0} & k_{x} n k_{0} & 0
\end{array}\right) .
$$

The following identities are used in derivations in the text:

$$
\begin{gathered}
P_{\gamma \alpha}(\mathbf{k}) P_{\gamma \beta}(\mathbf{k})=\delta_{\alpha \beta}-\frac{k_{\alpha} k_{\beta}}{k_{0}^{2}}, \\
P_{\alpha \gamma}(\mathbf{k}) P_{\beta \gamma}(\mathbf{k})=\delta_{\alpha \beta}, \\
T_{\gamma \alpha}^{\prime}\left(\mathbf{k}, \mathbf{k}^{\prime}\right) R_{\gamma \beta}\left(\mathbf{k}, \mathbf{k}^{\prime}\right)=\frac{-k_{z}^{\prime}}{k_{z}} R_{\gamma \alpha}^{\prime}\left(\mathbf{k}, \mathbf{k}^{\prime}\right) T_{\gamma \beta}\left(\mathbf{k}, \mathbf{k}^{\prime}\right), \\
R_{\mu \alpha}\left(\mathbf{k}, \mathbf{k}^{\prime}\right) R_{\mu \beta}\left(\mathbf{k}, \mathbf{k}^{\prime}\right)+\frac{\mathrm{A} 10)}{k_{z}} T_{\mu \alpha}\left(\mathbf{k}, \mathbf{k}^{\prime}\right) T_{\mu \beta}\left(\mathbf{k}, \mathbf{k}^{\prime}\right)=\delta_{\alpha \beta}-\frac{\tilde{k}_{\alpha} \tilde{k}_{\beta}}{k_{0}^{2}}, \\
R_{\alpha \beta}^{*}\left(\mathbf{k}, \mathbf{k}^{\prime}\right)=R_{\alpha \beta}\left(\mathbf{k}^{*}, \mathbf{k}^{\prime *}\right), \\
T_{\alpha \beta}^{*}\left(\mathbf{k}, \mathbf{k}^{\prime}\right)=T_{\alpha \beta}\left(\mathbf{k}^{*}, \mathbf{k}^{\prime *}\right) .
\end{gathered}
$$

Expressions (A9) and (A10) simply reflect the fact the $P$ is a projection operator with the usual properties that $P^{2}=P$ and $P$ is the identity on the subspace into which it projects. Expression (A11) may be understood to be a statement of Stokes reciprocity. 
[1] H. C. van de Hulst, Physica (Amsterdam) 15, 740-746 (1949).

[2] D. S. Jones, Philos. Mag. 46, 957 (1955).

[3] M. Born and E. Wolf, Principles of Optics, 7th ed. (Cambridge University Press, Cambridge, U.K., 1999).

[4] P. S. Carney, J. C. Schotland, and E. Wolf, Phys. Rev. E 70, 036611 (2004).

[5] L. Mandel and E. Wolf, Optical Coherence and Quantum Optics (Cambridge University Press, Cambridge, U.K., 1995).
[6] C. Tai, Dyadic Green's Functions in Electromagnetic Theory (IEEE Press, New York, 1994).

[7] A. A. Maradudin and D. L. Mills, Phys. Rev. B 11, 1392 (1975).

[8] P. S. Carney, E. Wolf, and G. S. Agarwal, J. Opt. Soc. Am. A 16, 2643 (1999).

[9] P. S. Carney, V. A. Markel, and J. C. Schotland, Phys. Rev. Lett. 86, 5874 (2001). 